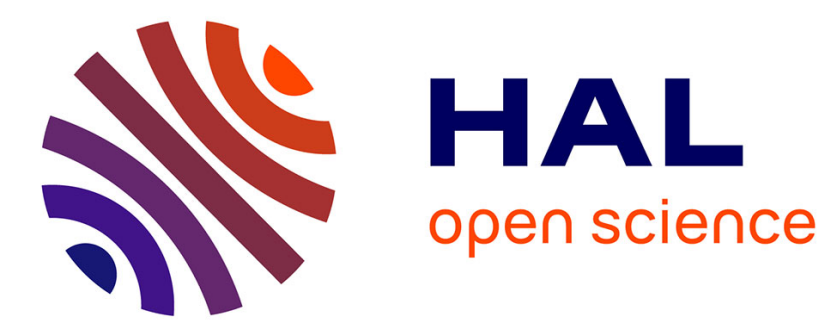

\title{
La direction d'un centre de recherches sociologiques dans la dictature chinoise
}

Jean Ruffier

\section{To cite this version:}

Jean Ruffier. La direction d'un centre de recherches sociologiques dans la dictature chinoise. Sociologies pratiques, 2014, Numéro Hors Série " Sociologies d'ici et d'ailleurs ", HS 1 (Supplément), pp.29-40. 10.3917/sopr.hs01.0029 . hal-01085228

\section{HAL Id: hal-01085228 \\ https://hal.science/hal-01085228}

Submitted on 13 Mar 2019

HAL is a multi-disciplinary open access archive for the deposit and dissemination of scientific research documents, whether they are published or not. The documents may come from teaching and research institutions in France or abroad, or from public or private research centers.
L'archive ouverte pluridisciplinaire HAL, est destinée au dépôt et à la diffusion de documents scientifiques de niveau recherche, publiés ou non, émanant des établissements d'enseignement et de recherche français ou étrangers, des laboratoires publics ou privés. 


\title{
LA DIRECTION D'UN CENTRE DE RECHERCHES SOCIOLOGIQUES DANS LA DICTATURE CHINOISE
}

\author{
Jean Ruffier
}

Presses de Sciences Po | Sociologies pratiques

\section{4/Supplément - HS 1}

pages 29 à 40

ISSN 1295-9278

Article disponible en ligne à l'adresse:

http://www.cairn.info/revue-sociologies-pratiques-2014-Supplément-page-29.htm

Pour citer cet article :

Ruffier Jean, «La direction d'un centre de recherches sociologiques dans la dictature chinoise », Sociologies pratiques, 2014/Supplément HS 1, p. 29-40. DOI : 10.3917/sopr.hs01.0029

Distribution électronique Cairn.info pour Presses de Sciences Po.

(C) Presses de Sciences Po. Tous droits réservés pour tous pays.

La reproduction ou représentation de cet article, notamment par photocopie, n'est autorisée que dans les limites des conditions générales d'utilisation du site ou, le cas échéant, des conditions générales de la licence souscrite par votre établissement. Toute autre reproduction ou représentation, en tout ou partie, sous quelque forme et de quelque manière que ce soit, est interdite sauf accord préalable et écrit de l'éditeur, en dehors des cas prévus par la législation en vigueur en France. II est précisé que son stockage dans une base de données est également interdit. 


\title{
La direction d'un centre de recherches sociologiques dans la dictature chinoise
}

\author{
Jean RUfFIER ${ }^{1}$
}

\section{Prologue}

Je suis entré dans la sociologie par l'étude des patrons français et l'observation participante des ouvriers avec l'équipe qui allait donnait naissance au Groupe lyonnais de sociologie Industrielle. J'ai appris des ouvriers que ces derniers en savaient toujours plus sur la production qu'on leur demandait, toujours plus que ce qu'on utilisait et que l'on pensait qu'ils savaient. Cela, je l'ai toujours vérifié dans tous les pays où j'ai pu travailler.

J'en ai gardé l'impression qu'un chercheur devait être capables d'appréhender tous les points de vue, surtout lorsqu'il y a affrontements et incompréhensions. Si l'on veut que la connaissance fasse bouger les choses, il faut qu'elle soit partagée par les acteurs. Donc une connaissance vraiment utile est une connaissance qui est cherchée en même temps par les chercheurs, les salariés et les cadres. II faut trouver les questions qui interrogent tout le monde à la fois. J'en ai gardé aussi une certaine modestie, on n'arrive pas à se mettre à la place de l'autre, qui nous reste fondamentalement incompris. Ce que la recherche peut apporter, ce sont des connaissances susceptibles d'aider les gens à travailler ensemble. Et puis j'entre au CNRS en 1979, à 30 ans, c'est formidable, j'acquiers la sécurité de l'emploi et de la fonction et d'un salaire qui permette de vivre. D'emblée, je décide d'être mon propre maître plutôt que de chercher l'approbation de la hiérarchie. Je vais faire les recherches que j'ai envie de faire et qui me semblent utiles. C'est mon côté caractériel.

Je suis "usinologue ", c'est-à-dire que je fais des recherches scientifiques sur les usines, comme d'autres en font sur les champignons. J'essaie de comprendre pourquoi et comment elles sont là et pas ailleurs, ce qui fait qu'elles vivent, se développent ou meurent. J'ai toujours été fasciné par les usines. II faut dire qu'une usine est une forme de miracle. Pour qu'elle vive, il faut que les humains qui sont dedans parviennent à maîtriser des techniques souvent complexes en se débrouillant au moins aussi bien que leurs concurrents. II leur faut aussi parvenir à produire des produits qui soient achetés par quelqu'un à des prix qui leur permettent de survivre. II leur faut enfin se faire accepter par les humains qui vivent autour, car, on le sait une usine est souvent aussi toxique, voire dangereuse pour ceux qui travaillent dedans, mais aussi ceux qui vivent à côté. Et ces maîtrises techniques et économiques doivent être atteintes par un groupe

1. Université Lyon 3. 
d'individus qui ont des intérêts divergents et beaucoup de raison d'entrer en conflit les uns avec les autres. II n'y a pas, disons plus, de laboratoire sur cette question en France et donc j'ai travaillé avec un réseau de chercheurs qui se posaient les mêmes questions que moi. J'ai beaucoup travaillé sur les transferts de technologie, car cela me permettait de trouver des financements et des gens intéressés par ces recherches, des chercheurs mais aussi des salariés et des dirigeants d'entreprises, ainsi que des fonctionnaires travaillant sur le développement industriel.

\section{Création du centre}

Je suis arrivé en Chine par hasard mais assez logiquement. Je cherchais un transfert de technologie où les interlocuteurs auraient le plus de mal possible à communiquer entre eux techniquement, linguistiquement, politiquement. Et voici qu'une société lyonnaise est envoyée aider la ville de Canton à concevoir son métro futur. Je vais voir le directeur de cette société et lui propose de suivre ce qui était sa première opération à l'export. Nous savions tous les deux que dans les échanges de technologies, la confiance est l'élément clé et donc je lui ai proposé de lui dire comment les Chinois les verraient. J'y mets deux conditions : qu'il me donne trois sous, et, que je puisse dire aux Chinois comment les Français les voyaient. L'accord est rapide et j'obtiens rapidement d'importants financements de recherches par le ministère des Transports qui y voit l'occasion de faciliter la vente d'un métro français. On est en 1988. Mes premiers contacts chinois m'amènent à m'appuyer sur le département de sociologie de l'Université Sun Yatsen ${ }^{2}$ à Canton.

Comme à mon habitude, j'ai pris soin de faire piloter la recherche par des Français et des locaux. La Chine est perçue comme une sorte d'ennemi par les autorités françaises et il m'est parfois difficile de faire accepter que mes informations sur les transferts de technologie soient partagées avec des chercheurs chinois. Et puis nous vivons les durs moments du printemps 89. Les Français et les Chinois sont impactés. Les Chinois qui étaient en France décident d'abandonner le projet pour ne pas avoir à retourner en Chine. Une partie des Français fait de même pour des raisons politiques. Je décide de rester : je savais déjà que la Chine était une dictature sanguinaire, j'avais engagé le projet en connaissance de cause. Les échanges de technologies se maintenaient, les collègues chinois de Canton désiraient ardemment que le projet se maintienne. Pour moi, qui avais construit mon travail sur la confiance, il était hors de question de laisser tomber mes camarades dans une situation délicate. Ce fut un moment difficile pour eux : le directeur du département de sociologie souhaitait que l'on remplace nos équipiers chinois par des gens plus proches de lui. Nous tenons bon, soutenant financièrement et humainement un collègue « envoyé à la campagne ". Ces épreuves ont permis aux uns de voir qu'ils pouvaient compter sur les autres. Une équipe est donc née ainsi. Le travail avance bien: nous repérons des difficultés de compréhension réciproques

2. L'Université Sun Yatsen est une université nationale installée à Canton. Elle est considérée comme la meilleure université du sud de la Chine (Hongkong excepté) et toujours classée parmi les 10 premières de Chine. 
entre les parties françaises et chinoises, mais nous repérons aussi les points forts qui permettent à quelques ingénieurs de travailler ensemble malgré la distance humaine entre eux. À ce moment, la France juge bon de vendre des avions de combat à Taïwan. Pékin interdit alors à Canton d'acheter un métro français. La Mairie de Canton décide malgré tout de conserver l'ingénierie française. Elle suit en cela les préconisations de nos rapports: il faut conserver les relations techniques qui marchent. Notre équipe franco-chinoise commence à avoir des alliés dans l'administration. Portés par ce succès, nos collègues chinois envisagent la création d'un laboratoire binational : ils craignent que l'université ne fasse éclater l'équipe car nombreux sont les universitaires qui souhaitent aller en France.

Des projets se succèdent, qui nous permettent de continuer à observer les entreprises de la région. Alors, nous décidons de faire une réunion d'un réseau international de chercheurs (l'INIDET ${ }^{3}$ ) à Canton, réunion à laquelle nous donnons la forme d'un colloque. Le département de Sociologie est ravi de cette initiative. Nous trouvons des sponsors dans les entreprises françaises sur lesquelles nous travaillons. D'anciens collègues chinois passés dans la sphère politico-administrative mobilisent les financements qui nous manquent pour faire de cette rencontre un événement visible. Nous décidons donc de monter en puissance et nous proposons à l'IRD ${ }^{4}$, qui s'intéresse depuis peu à la Chine, de s'impliquer dans le colloque et dans le projet de laboratoire. Le président de l'IRD viendra au colloque qui apparaîtra dès lors comme un événement politique pour l'administration chinoise. À la fin de ce colloque, l'université chinoise aura décidé de créer un centre de recherche franco-chinois et l'IRD d'y affecter un expatrié.

Fin 2000, le centre est inauguré, c'est une institution entièrement chinoise car elle a le statut d'un centre de recherche ordinaire de l'université. Son originalité est d'avoir été inauguré par un consul français et d'avoir deux directeurs, un Chinois et un Français.

L'IRD expatrie Rigas Arvanitis à Canton. Pendant près de cinq ans Rigas sera le principal permanent du centre. II lui donnera vie et accueillera de jeunes chercheurs, mènera des projets de recherches internationaux et aidera l'Université Sun Yatsen à obtenir une chaire de l'UNESCO.

Le CNRS accordera un projet de recherches international au Centre franco-chinois de recherches en sociologie de l'industrie et des technologies. Des accords ont été signés entre les universités lyonnaises Lyon 2 et Lyon 3.

En 2008, le CNRS décide de m'expatrier à Canton pour relancer le centre. L'expatriation durera deux ans puis s'arrêtera faute de financement, le CNRS et le MAE réduisant considérablement leur voilure à l'étranger. Le centre continue à exister mais son activité est réduite aux projets qui passent par lui.

3. L'Institut international pour le développement des technologies (INIDET) est en fait un réseau informel constitué par une quinzaine de chercheurs de différents pays. Pendant une quinzaine d'années, ce réseau aura fonctionné comme un véritable laboratoire organisant des réunions régulièrement (malgré le coût de nombreux billets transcontinentaux) et définissant des projets de recherches communs qui ont notamment conduit à mettre au point le concept et les instruments de mesure de l'efficience productive.

4. L'Institut de recherches pour le développement est un organisme de recherches français, spécialisé sur les pays en voie de développement. 


\section{La direction d'un centre chinois}

Le fait de créer et prendre la direction d'un centre de recherches sociologiques dans une université d'État chinoise n'allait pas de soi. C'était même un paradoxe dans un pays où la loi précise que les étrangers ne peuvent pas faire de recherche en sciences sociales. Le président de l'Université Sun Yatsen a donc pris un risque. Comme toujours en Chine, on va jouer sur le flou: bien que nommé directeur d'un de ses centres de recherches, je n'avais aucun statut dans l'université. Cette situation a perduré jusqu'à aujourd'hui et à deux reprises j'ai appris que ma nomination avait été renouvelée sans d'ailleurs que je sois informé d'une quelconque périodicité des nominations.

Pendant des années, je suis venu à Canton avec un visa de touriste, et j'ai animé le centre, lancé des projets de recherches, formé des chercheurs, fait des enquêtes, aidé des jeunes ou moins jeunes chercheurs à mener des enquêtes sur la région cantonaise.

Deux autres colloques internationaux ont pu être organisés en 2004 et 2010, ils ont contribué à rendre plus visible son existence et lui donner un peu plus de rayonnement. II reste que Canton est un lieu différent de Pékin ou Shanghai : la recherche y est plus facile. En effet, la plupart des chercheurs expatriés occidentaux sont envoyés à Pékin. Cela a une certaine logique car Pékin rassemble les intellectuels chinois les plus connus et donc les plus reconnus. S'y trouve aussi l'ambassade de France qui se nourrit de la production des intellectuels français. Enfin, les principales agences de financement de la recherche s'y trouvent également. Mais Pékin a un gros défaut : c'est le lieu du pouvoir et les chercheurs en sciences sociales y sont pris entre une double contrainte, ne pas attirer les foudres du pouvoir chinois, ne pas déranger l'action diplomatique de l'ambassade. Cela ne serait pas très gênant si la Chine était un État de droit et si les intellectuels français étaient indifférents à la répression politique qui y sévit. Lorsqu'ils veulent engager des travaux de terrain en s'appuyant sur une organisation chinoise, leurs partenaires chinois doivent, comme à Canton, demander l'autorisation. Cette autorisation est rarement refusée, mais elle n'est pas non plus accordée car il y a toujours un élément de la conjoncture qui rend l'enquête problématique aux yeux de l'appareil de sûreté chinois.

Shanghai se trouve logée à la même enseigne que Pékin pour la recherche en sciences sociales. Shanghai a pour mission d'attirer le siège des multinationales étrangères. On y rend donc la vie plus agréable aux expatriés en insistant sur les aspects culturels et économiques. Mais en même temps, les autorités y sont très sensibles à tout ce qui peut faire tache et les chercheurs français font souvent tache. Canton, de ce point de vue se trouve dans une situation vraiment différente. Canton est au centre de la région la plus productrice de devises. Cette région a depuis longtemps bénéficié d'un régime spécial, on y laisse les acteurs sociaux plus libres de manière à ne pas entraver l'efficacité économique. Cela vaut pour l'université. Les débats y sont plus ouverts, les autorisations de recherches plus faciles même quand elles impliquent des chercheurs étrangers. En fait, paradoxalement, il est presque plus facile pour moi de faire des observations d'usines en Chine qu'en France. Les entreprises me sont très accessibles : si ce sont des entreprises étrangères, les cadres expatriés sont toujours heureux de rencontrer un occidental avec lequel discuter. Si ce sont des entreprises privées chinoises, les patrons ont très envie de bénéficier de notre expertise dans la mesure où nous comparons toujours les usines visitées avec celles vues ailleurs, et donnons généralement un diagnostic sur les points faibles et les points forts quant à la 
survie dans la durée de l'usine observée. Si c'est une entreprise d'État, c'est encore plus simple car l'autorisation de faire l'enquête devient pratiquement une injonction à en faciliter le déroulement. Malgré de faibles moyens budgétaires et des séjours souvent courts, nous avons pu maintenir un suivi du tissu industriel régional.

\section{Le consensus intellectuel chinois}

La Chine dispose d'une forme de pouvoir qui a peu d'équivalents ailleurs ou dans le passé. II s'agit non pas d'une monocratie mais d'une oligarchie ${ }^{5}$. En fait, une des caractéristiques de l'oligarchie chinoise et d'avoir résolu la plupart des conflits en son sein de manière non mortelle ${ }^{6}$. C'est un petit groupe de dirigeants, constitué de rescapés de la longue marche, qui a pris le pouvoir en 1949. Ce petit groupe constitue une petite société qui se reproduit en son sein et conserve depuis plus de soixante ans les rênes du pouvoir. Les observateurs étrangers s'entendent en général pour dire que l'on sait très peu de choses sur la vie de ces quelques deux mille personnes qui constituent le groupe dirigeant, sinon que les décisions essentielles sont prises par un collectif aux contours mal connus et que ce collectif tient fermement les rênes d'un parti communiste de plusieurs dizaines de millions de personnes. L'oligarchie permet de résoudre le problème de la transmission du pouvoir d'une génération à l'autre. Les mêmes personnes qui sont responsables des erreurs de la planification initiale, puis des crimes de la RévoIution culturelle, sont aujourd'hui celles qui dirigent la politique dite de socialisme de marché ou d'ouverture. Ce mode unique de gouvernement oligarchique explique probablement la longévité exceptionnelle du régime. On mesure mieux le pouvoir de cette oligarchie quand on constate que $98 \%$ des milliardaires chinois sont apparentés à des personnes qui tiennent ou ont tenu des fonctions ministérielles. L'avantage du groupe sur la personne, c'est que lorsque les erreurs du dirigeant sont visibles, il se trouve souvent dans le groupe des gens qui ont déjà un plan pour corriger les effets de ces erreurs et proposer des solutions alternatives. Les dirigeants chinois ne sont pas plus intelligents que les dirigeants des autres pays, mais leur système est plus "pardonnant ", c'est-à-dire qu'il corrige au fur et à mesure les erreurs des dirigeants. Et la durée du collectif est potentiellement infinie. Le pouvoir central ne travaille pas pour la postérité, comme le ferait un potentat qui sait qu'il va mourir ; il ne travaille pas non plus pour aligner des résultats à la date des élections suivantes, puisqu'il n'y a pas vraiment d'élections. II a la possibilité et l'intérêt de se projeter et de projeter son pays dans le long terme. Cela explique le développement rapide des infrastructures, la priorité absolue mise sur l'unité du pays et la non mise en cause de son système politique.

La société chinoise est devenue une composante de la société globale. L'usine du monde n'est pas qu'une image, c'est la constatation que l'économie mondiale ne saurait aujourd'hui fonctionner sans la Chine. Beaucoup de pays ont délocalisé une partie si importante de leur production en Chine qu'ils seraient bien mal à l'aise si les usines

5. Oligarchie : gouvernement par un petit nombre de personnes, se différencie de la monarchie, gouvernement d'un seul, et de la démocratie, gouvernement par le peuple.

6. À l'exception notable de Lin Piao, successeur désigné de Mao. 
chinoises s'arrêtaient de produire. Pays très dépendant de son commerce extérieur, la Chine n'en est pas moins un pays qui refuse d'appliquer les règles communes. Ainsi garde-t-elle une monnaie non convertible, donc une monnaie dont l'appréciation n'est pas directement fixée par le marché. Si les Américains se plaignent volontiers de la valeur trop basse du yuan, on doit admettre que le système financier chinois a joué un rôle important dans l'arrêt de la crise asiatique ou des deux dernières crises bancaires. Refusant de soumettre sa monnaie aux lois du marché, l'État chinois peut agir sur une partie significative de la monnaie mondiale. Si certains économistes dénoncent ce qu'ils appellent une tricherie, d'autres se réjouissent de constater que malgré le libéralisme dominant, il existe des leviers d'action sur la monnaie et l'économie mondiale qui dépendent de volontés politiques, fussent-elles chinoises.

L'État chinois reste très interventionniste et planificateur. L'idéologie socialiste a été un peu rapidement enterrée en Occident, lors de la désagrégation de l'URSS. La planification a été abandonnée dans nombre de pays, parce que l'idéologie du pouvoir était favorable au laisser-faire/laisser-aller, ou parce que le gouvernement n'avait plus les capacités de dicter ses instructions aux entreprises. L'État chinois reste au contraire capable de planifier, il peut mobiliser rapidement une partie importante des richesses nationales pour faire face à l'imprévu. L'État chinois garde une prérogative qu'avaient les grands États jusque vers la fin du $x x^{\circ}$ siècle : il peut décider qui a le droit d'être riche parmi ses citoyens. L'État continue à penser pour ses citoyens à leur place, il continue à peser sur leur vie quotidienne, décrétant combien d'enfants ils doivent avoir, s'ils peuvent ou non sortir du pays, etc.

Les intellectuels et responsables politiques chinois ont gardé en mémoire l'effondrement brutal de l'URSs. II y a une forme de consensus en Chine, consensus entre le pouvoir et ceux qui contestent son hégémonie sur la société chinoise: les Chinois veulent absolument éviter de vivre ce qu'a vécu l'uRss à la fin du dernier siècle. II existe donc bien une forme de consensus des intellectuels et du pouvoir dans l'idée qu'il faut éviter le chaos que ne manquerait de produire un changement politique majeur. Ce consensus explique une certaine liberté de débat dans la société chinoise. II ne se passe pas une semaine sans que tel ou tel aspect de la politique gouvernementale ne soit mis en cause par un intellectuel ou un haut cadre. Dans l'université, les débats sont très libres. Mais les limites de cette liberté apparaissent facilement à quiconque : il suffit de voir qui est emprisonné. On peut être un intellectuel qui discute ouvertement de l'évolution de la société chinoise et des politiques mises en œuvre et rester en liberté à trois conditions :

- ne pas exposer la Chine à la critique de l'étranger ;

- ne pas mettre en cause l'intégrité territoriale chinoise ;

- ne pas créer de nouvelles organisations politiques ou syndicales.

Aller à l'encontre de ce consensus s'avère très risqué pour des citoyens chinois, ce qui n'empêche pas certains d'entre eux de continuer à défier le pouvoir. Ceux-là sont une très petite minorité dont le courage force l'admiration. La situation intellectuelle chinoise est très différente de celle qui prévalait dans les pays socialistes de l'Est européen. La plupart des intellectuels y avaient pris l'habitude de masquer leurs opinions en public. Les publications étaient généralement encadrées d'une introduction et d'une conclusion qui reprenaient l'idéologie officielle et n'avait que fort peu à voir avec le reste du contenu. On trouve peu cela en Chine. Les professeurs et les chercheurs disent 
assez facilement le fond de leur pensée devant des collègues chinois ou des officiels. Et ils sont très critiques avec l'idéologie officielle du parti, et n'hésitent pas à s'amuser de la paranoïa du pouvoir ou à critiquer la corruption des fonctionnaires. On pourrait dire qu'ils sont peu perméables aux campagnes idéologiques. Mais ils restent strictement à l'intérieur du consensus soit qu'ils sont intimement en accord avec ce consensus, soit qu'ils ne voient pas l'intérêt d'une rupture au regard des risques qu'elle leur ferait prendre.

\section{Analyse comparative plutôt qu'analyse culturelle}

La Chine présente des éléments de spécificité sociétale évidents, peut-être trop évidents. D'une part, il s'agit d'un immense pays, pratiquement le cinquième de l'humanité, et d'un pays qui est assez largement fermé sur lui-même. Il est des périodes de l'histoire de la Chine impériale où les étrangers n'avaient pas le droit de résider en Chine, des périodes où la sortie du territoire national était punie de mort. Dans la période moderne et jusqu'aux alentours de 1995, les étrangers allaient assez difficilement en Chine, leurs déplacements et lieux de résidence étaient très contrôlés. Aujourd'hui, la situation a largement changé et le pays accueille de nombreux étrangers de nombreux pays ayant une grande variété d'occupations et de contacts avec les citoyens chinois. II n'en reste pas moins qu'ils doivent fréquemment renouveler leur titres de séjours et que leurs lieux de résidence sont contrôlés, que certaines zones sont en permanence ou occasionnellement inaccessibles. Les communications avec l'étranger et sur le territoire national sont l'objet de surveillances et de censures. De nombreux Chinois vont à l'étranger pour le travail ou les études : ils contribuent à une forme d'ouverture qui semble irréversible mais reste limitée au regard de l'immensité de la population.

L'autre aspect de la spécificité chinoise tient à une langue non alphabétique. II s'agit d'une rareté sur notre planète et à l'évidence cette forme de langue structure la pensée d'une manière différente. Pour faire très court, une langue alphabétique se lit linéairement, comme un discours parlé et pousse à une causalité temporelle, le présent s'expliquant par le passé, la fin arrivant après le début. Une langue non alphabétique peut se lire comme un paysage, sans suivre un ordre préconstruit. On peut opposer l'explication par l'harmonie à la causalité temporelle : s'il constate un point chaud, l'Occidental cherchera ce qui a chauffé dans le passé ce point, le Chinois cherchant le froid qui contrebalance ce chaud dans une vision causale synchronique. À bien y regarder, les deux modes sont aussi scientifiques et aussi explicatifs de la réalité l'un que l'autre.

Faut-il pour autant adopter des méthodes spécifiques pour analyser la Chine ? C'est le point de vue des analyses culturalistes, analyses qui renvoient la spécificité des faits sociaux observés en Chine à la culture chinoise. Sans rejeter l'intérêt de travaux culturalistes, nous pensons difficile d'appuyer une théorie scientifique sur une démarche comparative qui présuppose la spécificité du contexte. En ce moment par exemple, nous cherchons précisément à voir si les mêmes raisons qui ont amené à la disparition du taylorisme en France vont jouer en Chine. II nous faut donc imaginer qu'un ouvrier chinois ressemble à un ouvrier français. C'est bien sûr très difficile si l'on se focalise sur la langue et la culture de ces deux populations. Cela l'est un peu moins si l'on met les uns et les autres en situation en supposant que mis dans la même situation, disposant 
des mêmes ressources, les ouvriers chinois vont plus ou moins se comporter comme les ouvriers français. Dans nos enquêtes, nous privilégions systématiquement l'analyse des situations sur le recours à l'explication culturelle. L'explication culturelle nous semble souvent trop confuse, dans la mesure où nous travaillons sur des populations aux origines géographiques et sociales variées (patrons, fonctionnaires, ouvriers paysans). Souvent, la langue employée manque de finesse car les entretiens se font sans utiliser la langue maternelle de l'interviewé. J'ai pu me rendre compte que les parlers locaux sont très nombreux, même dans le Guangdong, il est fréquent que les Cantonais ne comprennent pas la langue d'autres Cantonais résidant à une vingtaine de kilomètres les uns des autres. Si le communisme a visé l'homogénéisation de la langue et de la culture, il a surtout mis la confusion dans les langues parlées et les cultures de référence, si bien qu'il est toujours hasardeux de renvoyer un comportement à une culture donnée. Tout le monde dispose d'une langue commune, mais peu la maîtrisent correctement. Chez les intellectuels, la maîtrise de la langue chinoise se limite aux domaines de spécialisation de la personne en question ${ }^{7}$. Quant aux références culturelles communes, elles renvoient à une doxa à laquelle tout le monde peut peu ou prou se référer, mais qui en fait ne correspond pas aux pensées intimes de chacun.

Les difficultés linguistiques que nous avions dans nos premières missions chinoises nous ont poussé à utiliser la mise en situation pour interpréter les positions et les discours. Nous avons assez rapidement compris que cette méthode nous permettait de meilleurs contacts avec nos interlocuteurs que le passage par des traducteurs de haut niveau. Lorsque l'on se trouve devant un interlocuteur qui prend une position qui nous étonne, il est souvent plus rapide de chercher l'explication de cette position dans la situation qu'il vit et les informations et ressources qu'il a, en se demandant si dans la même situation et avec les mêmes moyens on ne serait pas tenté de recourir à la même position. Renvoyer cette position à la soi-disant culture particulière de cette personne est risqué dans la mesure où, bien souvent, dans une même usine, on trouve des personnes de cultures fortement différentes les unes des autres. De plus, travailler sur les situations permet la falsification ${ }^{8}$, si l'on définit une situation corrélée à certaine position, on peut se mettre en mesure de travailler de manière scientifique, de recourir à une démarche que les non spécialistes de la Chine (mais peut-il y avoir vraiment de réels experts de la Chine ?) puissent comprendre et critiquer. L'analyse comparative est simplificatrice mais elle permet le débat avec une large communauté scientifique, et

7. La langue chinoise n'est pas une langue alphabétique. Il est pratiquement impossible de comprendre un mot nouveau en regardant seulement comment se mot est construit. Ainsi chaque fois qu'un scientifique essaie de préciser sa pensée à travers un mot existant ou un mot qu'il crée, il génère un nouveau sens qui ne peut être découvert qu'en lisant un lexique spécifique, ou en passant par une langue étrangère. Ainsi les concepts des grands penseurs occidentaux sont traduits par des caractères spécifiques qu'il faut apprendre avant de pouvoir parler de ces penseurs avec des érudits chinois.

8. Thomas Kuhn propose la falsification comme moyen de vérifier la validité scientifique d'une proposition. Lorsque le scientifique émet une théorie, il a tendance à ne voir dans la réalité que ce qui conforte sa théorie ou son hypothèse. La démarche de falsification consiste à ne pas chercher à démontrer ce que le scientifique pense vrai mais à se demander quel test convaincant il pourrait effectuer qui, s'il était positif, lui prouverait qu'il se trompe. Lorsque j'essaie de tester une hypothèse, j'écris cette dernière de manière que, pour moi, sa vérification ou son invalidation soient toutes deux acceptables. Peu m'importe que mon hypothèse soit juste, ce qui importe c'est que le travail que je fais sur elle me permette de faire avancer la connaissance. 
malgré ces approximations, elle a largement contribué à améliorer notre perception de l'industrie chinoise.

\section{Dire le vrai face à la censure}

Vers la fin 2000, le président de l'Université Sun Yatsen à Canton a décidé de créer un centre de recherches en sciences sociales et m'en a nommé directeur français. Dès lors me voilà devenu une sorte de mandarin faisant de la recherche dans une université qui ne me paie pas mais autorise de fait les recherches que je fais, et autorise les chercheurs que je choisis à les faire avec moi. Bien sûr, il y a des contraintes, mais elles ne sont pas explicites. La loi chinoise interdit aux étrangers de faire de la recherche en sciences sociales. Autrement dit, je n'ai aucun droit, juste des tolérances. Si le centre dispose de bureaux indiqués d'une plaque en cuivre écrite en chinois et en français, comme directeur, je n'apparais pas dans l'annuaire téléphonique. Je dois donc avancer avec précaution, et surtout, je ne veux pas mettre en difficulté les chercheurs chinois qui travaillent avec moi. Ils choisissent librement de le faire. Si ce que je publie pose un problème de sécurité publique, ils risquent de se voir accuser de trahison. II ne s'agit pas d'un cas d'école. Des chercheurs chinois se sont trouvés emprisonnés à la suite de publications par des étrangers d'enquêtes auxquelles ils avaient contribué. II est des sujets que je sais ne devoir jamais aborder. Je ne dois jamais mettre en cause l'intégrité du territoire chinois, ni la suprématie du Parti. Mais, ma position m'ouvre un accès à des informations sur les entreprises que je n'aurais pas autrement. J'ai pu faire bénéficier de nombreux collègues de cet avantage et ainsi, nous avons contribué à mieux comprendre comment le sud de la Chine était devenu l'atelier du monde.

II me faut dire deux mots sur l'autocensure. J'ai choisi de ne jamais mentir, c'està-dire de ne jamais écrire quelque chose que je sais faux. Cela n'est pas vraiment difficile. Plus délicate est la question de la critique. Un chercheur, surtout un chercheur en sciences sociales, produit un discours critique par définition, puisqu'il interroge les rapports dominants/dominés. Et donc mon discours sur la politique industrielle chinoise est souvent critique. Les autorités chinoises ne tireraient aucun bénéfice d'un chercheur étranger qui ne leur montrerait pas leurs faiblesses. Je prends garde de donner la primeur de ces critiques aux collègues chinois. Ils savent ainsi à quoi s'en tenir et peuvent critiquer mes critiques avant une diffusion plus large. Bien sûr, il est toujours un peu frustrant de voir des collègues montrer les faiblesses d'une conclusion et d'une observation. Leur refus de cosigner ce que j'écris équivaut à une interdiction de publication. Mais cela, tout chercheur scientifique y est habitué. C'est la discussion entre scientifiques qui est le moyen de valider une observation, une déduction. Les scientifiques sont donc habitués à devoir reprendre un texte qu'ils pensaient achevé, et il faut parfois très longtemps pour parvenir à un consensus entre chercheurs participant du même projet. De ce point de vue, la situation chinoise n'est pour moi pas différente de la situation française : entre chercheurs d'un même projet il faut se mettre d'accord sur les questions que nous posons, sur le contenu des observations que nous faisons et sur les conclusions que nous en tirons. Et le désaccord signifie généralement une faiblesse que l'on doit corriger avant toute diffusion hors de l'équipe de recherche. On peut imaginer que les chercheurs chinois s'autocensurent plus que les chercheurs français, 
mais cette autocensure leur coûte autant, sinon plus qu'aux chercheurs français. Ils vont chercher les moyens de rendre diffusables nos observations, et en fait, si j'ai eu souvent à reprendre des textes, je ne crois pas avoir écrit des textes que je désapprouverais aujourd'hui. Je n'ai jamais abandonné la diffusion d'un résultat qui s'est avéré bien établi. II faut dire que je travaille sur des sujets qui sont acceptés par les autorités chinoises, en quelque sorte la censure se fait sur les sujets plus que sur la méthode.

Le problème est ailleurs. Je suis devenu une sorte d'expert de la Chine, même si la Chine est pour moi un terrain plus qu'un objet de recherches. Et de fait, on me demande souvent mon opinion sur ce qui se passe en Chine, dans des sujets qui ne sont pas de ma compétence. L'exemple le plus frappant est celui de la situation au Tibet. Pratiquement tous mes amis ont un avis sur la question tibétaine. En général, les Français pensent que l'État chinois a comme volonté de faire disparaître la culture tibétaine et qu'il vaudrait mieux que le Tibet soit indépendant ou autonome. Et on me demande mon avis. Si je m'avisais à me ranger à un tel jugement, pour les autorités chinoises, je franchirais une ligne rouge. Cela pourrait nuire à ma capacité à faire des séjours en Chine. Cela pourrait mettre en grande difficulté mes amis et collègues chinois qui se verraient probablement reprocher leur collaboration avec quelqu'un qui met en danger l'intégrité du territoire chinois. Je connais des amis français, résidant en Chine, qui ont pris le parti de reprendre à leur compte la position gouvernementale chinoise. Cela leur assure une tranquillité face aux autorités, et parfois une réelle popularité parmi les Han, population qui représente plus de $80 \%$ de la population résidente en Chine. Personnellement, je ne suis pas certain que cette position me conviendrait. Je sais le sujet délicat, source de conflits et de drames profonds. Un scientifique qui veut travailler scientifiquement sur une question se doit d'être indifférent aux résultats de ces recherches : donc si je travaillais sur le sujet, je ne saurais pas à l'avance la position qui sera la mienne à l'issue de la recherche. Décider de ne pas mentir rend impossible de moduler mon opinion en fonction de mes interlocuteurs. Je ne suis pas certain que ce que j'affirme à l'issue de mes recherches est juste, aussi ne vais-je pas affaiblir ces dernières en les modifiant constamment. Donc, j'ai pris soin depuis longtemps de tenir le même discours où que je sois. II faut dire que depuis que je travaille à l'étranger, j'ai toujours constitué des équipes internationales, et fait en sorte que me collègues étrangers aient l'occasion de comparer ce qu'ils voyaient et entendaient chez eux avec ce qui se passe et se dit en France. Et donc je ne peux pas tenir à ces collègues des discours différents suivant le lieu où je me trouve. Je n'ai d'ailleurs pas de talent pour la schizophrénie, il m'est vraiment plus simple de tenir un seul discours. C'est pourquoi, sur la question tibétaine, j'ai finalement décidé que je ne m'intéresserais pas à cette question et que par conséquent, je ne serais jamais à même d'avoir une opinion étayée sur le sujet. II est vrai que la question est complexe et il est difficile de dire si les Tibétains seraient plus ou moins heureux dans un État tibétain. En tout cas, moi, je n'ai pas travaillé sur cette question et donc ne peux y répondre. Je suis dans la position un peu particulière d'un spécialiste qui serait incompétent là où le profane prend parti. Voici donc la position que j'ai choisie, me rendre incompétent dans certains domaines pour n'avoir ni à me renier, ni à risquer de déclencher des réactions non souhaitées. En fait, je ne pourrais pas travailler de manière scientifique sur le sujet de l'indépendance tibétaine, en tout cas pas avec ma méthode qui suppose un consensus des chercheurs sur ce qu'on doit publier. 
Je garde le même discours et les mêmes réserves, que je sois en Chine ou ailleurs. Pour moi, un scientifique se doit d'une certaine cohérence, il n'est pas question pour moi que le lecteur regarde où j'ai écrit tel ou tel papier pour savoir s'il est scientifique ou non. En fait, je travaille en Chine comme en France, avec des données différentes. J'essaie de confronter mes observations avec les observations de mes collègues, qu'ils soient chinois ou français ou encore d'autres pays. Je veille à soumettre à la critique de mes collègues toutes les analyses que je peux produire. Le fait d'être en Chine modifie les sujets que j'ai la possibilité de travailler sérieusement, mais pas ma démarche scientifique. Je sais que le fait d'avoir pris une responsabilité dans l'administration chinoise de la recherche me conduit à respecter certaines limites, mais je trouve que les données que je récolte sont suffisamment précieuses pour que cela justifie mon investissement personnel et les deniers que l'État français engage dans les recherches auxquelles je participe.

Je sais très bien pourquoi je suis en Chine : tenter de définir les ressorts d'un développement industriel autonome. Pour cela, il me faut disposer du plus possible d'informations fiables sur ce qui se passe dans l'industrie chinoise. Si je me suis empêché de devenir un spécialiste de la Chine, j'ai recueilli des informations qui n'étaient pas autrement disponibles. Je crois ainsi avoir rendu davantage service à la communauté scientifique en apportant des éléments nouveaux qu'en participant à tous les débats. J'ai un questionnement qui rejoint assez facilement celui des acteurs économiques. Je me demande comment on a réussi ce qu'on a réussi et comment on peut faire mieux, ou éviter d'aller plus mal. Posant les mêmes questions qu'eux, je travaille avec eux à y répondre. Ils comprennent vite que nous n'avancerons que si nous travaillons sur des données réelles. Mon accès au terrain se doit à cela. II a un certain coût mais je trouve que cela en vaut la peine.

\section{Conclusion}

J'ai une idée assez précise de pourquoi j'ai créé ce centre en Chine, les avantages de cette position et les contraintes. Je vais commencer par les contraintes : je ne peux pas parler de tout ce que je pense sur la Chine en public. Dès que je parle de la Chine, je suis un mandarin pris dans les contradictions du régime chinois. Je respecte donc absolument le consensus dont j'ai parlé plus haut. Je ne critique donc pas le gouvernement chinois pour sa politique des droits de l'homme ou son action au Qingjiang ou au Tibet. Je laisse cela à d'autres. Je ne m'interroge sur le système politique que pour ce qui concerne sa politique industrielle. Ma recherche en Chine est scientifique. Le but de la science pour moi, est le même que celui que se fixait Descartes : il s'agit d'augmenter la durée de la vie en bonne santé de chacun. Ma démarche est scientifique et elle procède selon une méthode participative. Je m'interroge avec chacun des acteurs avec lesquels je travaille (ouvriers, cadres, patrons, fonctionnaires) sur la manière de faire en sorte que le développement industriel ne se fasse pas au détriment des uns ou des autres. Autrement dit, je les fais travailler sur mes hypothèses de recherches et je recueille les informations dont j'ai besoin en utilisant toutes les méthodes appropriées pour cela. Ainsi mon but n'est pas opposé à celui des autorités chinoises, non que je cherche à leur faire plaisir, mais ma méthode de travail ne permet pas de poursuivre 


\section{LES SOCIOLOGUES DANS DES ESPACES EN TRANSITION}

un but secret. C'est une limite. Par contre, ce but me permet de recueillir des informations dont les chercheurs ne disposent généralement pas sur le système industriel chinois. Si mes contacts sont persuadés que notre travail peut leur être utile, ils auront tendance à nous donner des informations justes pour avoir des conclusions justes. Ainsi j'ai pu travailler sur les grèves en Chine du Sud. Je l'ai pu car il y avait un véritable débat au sein des autorités sur le pourquoi de ces grèves et les options à prendre pour y faire face. J'ai pu ainsi voir des oppositions et des stratégies différentes chez les membres du pouvoir politique et de la hiérarchie syndicale. J'ai pu montrer que ces grèves n'étaient pas près de s'arrêter et qu'elles sont cause et conséquence de grands changements dans les attitudes de la main d'œuvre. Pour faire ce travail, j'ai donc profité d'une ouverture, d'une fenêtre de débats qui semble fermée aujourd'hui, mais se rouvrira, espérons-le, bientôt. J'ai créé ce centre pour précisément faire exister un lieu de débats scientifiques sur le monde de l'industrie entre chercheurs chinois et chercheurs occidentaux. Nous profitons d'un espace limité, mais nous en profitons. 